\title{
Urgences
}

\section{Le tour de l'île de Félix Leclerc}

\section{André Gaulin}

Numéro 26, décembre 1989

Des textes qui chantent

URI : https://id.erudit.org/iderudit/025554ar

DOI : https://doi.org/10.7202/025554ar

Aller au sommaire du numéro

Éditeur(s)

Urgences

ISSN

0226-9554 (imprimé)

1927-3924 (numérique)

Découvrir la revue

Citer cet article

Gaulin, A. (1989). Le tour de l'île de Félix Leclerc. Urgences, (26), 47-55.

https://doi.org/10.7202/025554ar d'utilisation que vous pouvez consulter en ligne.

https://apropos.erudit.org/fr/usagers/politique-dutilisation/ 


\section{Le tour de l'île de Félix Leclerc André Gaulin}

\section{Le tour de I'Tle}

(Introduction musicale)

I Pour supporter le difficile

et l'inutile

$Y$ a l'tour de l'île

quarante-deux milles

5 de choses tranquilles

Pour oublier grande blessure

dessous l'armure

été hiver

y a l'tour de l'île

10 l'île d'Orléans.

II L'île c'est comme Chartres

c'est haut et propre

avec des neis

avec des arcs des corridors

15 et des falaises

En février

la neige est rose

comme chair de femme

et en juillet

20 le fleuve est tiède

sur les battures.

III Au mois de mai

à marée basse

voilà les oies.

25 Depuis des siècles

au mois de juin

parties les oies.

Mais nous les gens

les descendants de La Rochelle

30 présents tout l'temps

surtout l'hiver

comme des arbres.

Mais c'est pas vrai

ben oui c'est vrai

écoute encore. 
(llot sonore)

IV Maisons de bois maisons de pierre clochers pointus et dans les fonds

40 des pâturages de silence des enfants blonds nourris d'azur comme des anges jouent à la guerre imaginaire.

$\checkmark 45$ Imaginons

lîle d'Orléans

un dépotoir

un cimetière parc à vidanges

50 boîte à déchets U.S. parking.

On veut la mettre en mini-jupe and speak english.

Faire ça à elle

55 l'île d'Orléans notre fleur de lyse

Mais c'est pas vrai ben oui c'est vrai raconte encore.

VI 60 Sous un nuage près d'un cours d'eau c'est un berceau et un grand-père au regard bleu qui monte la garde.

II sait pas trop

$65 \mathrm{c} \theta$ qu'on dit dans les capitales l'œil vers le golfe où Montréal guette le signal.

Pour célébrer l'indépendance

70 quand on y pense c'est-y en France c'est comme en France le tour de l'île quarante-deux milles

75 comme des vagues des montagnes 
les fruits sont mûrs

dans les vergers

de mon pays.

VII 80 Ça signifie

l'heure est venue

si t'as compris...

(Conclusion musicale)

Félix Leclerc, Le tour de l'île ${ }^{1}$,

Philips, 6325142

Aux deux bouts de l'aventure chansonnière de Félix Leclerc, deux chansons témoignent de son long cheminement. En 1934, c'est un jeune homme de vingt ans qui écrit Notre sentier, une chanson que Jacques Canetti trouve trop triste pour suggérer à Leclerc, venu en France, de l'inclure à son répertoire d'à peine trente chansons, pourtant. Effectivement, on ne retrouve cette première chanson ${ }^{2}$ gravée sur disque qu'après $1960^{3}$. La chanson de $1934^{4}$ lie Leclerc à la tradition médiévale par la présence

1 Texte tiré du livre du même titre, Montréal, La courte échelle, 1980, [n.p.].

2 La deuxième chanson Sur le bouleau, tres brève, date de 1937; la troisième, Un petit soulier rose, de 1940, plus personnelle; la quatrième, de 1943, humoristique et significative de la poétique du chansonnier, Le Québecquois, propose déja un modèle que fonde le regard. C'est en 1944 que les chansons vont se multiplier, la seule année 1946 en comptant neuf, l'année 1948 en donnant quatre qui sont toujours restées liées au répertoire du chansonnier. Cette lente inscription de Leclerc dans le genre manifeste sans doute à la fois son hésitation à participer de cette manière à l'écriture, mais aussi témoigne d'un a horizon d'attente " quasiment inexistant.

3 En tout cas, ni le Félix Leclerc ( $\Gamma^{\circ}$ 1) chante ses demiers succes sur disque (Philips, B.76.087 R), ni le Fólix Leclerc et sa guitare nீ 2 (Epic LF. 2008), ni le Fúlix Leclerc et sa guitare to 3 (Epic LF. 2012) ne donnent cette chanson. Pas plus d'aitleurs qu'aucun des trois premiers microsillons faits chez Philips en 1953 (Moi, mes souliers), en 1957 (La drave), en 1960 (L'heritage). II faut attendre Le roi heureux, en 1962, pour voir figurer pour la premiàre fois Notre sentier. Puis, en 1967, dans l'album double Pleins feux sur Félix Leclerc (i paraît deux albums doubles du même titre cette même année, des réalisations de Philips 6641.030 et 6641.135 , distribués par London Records of Canada Ltd), Michel Legrand orchestre Notre sentier, une chanson d'une durée de deux minutes trente-huit qui en deviendront trois minutes cinquante et une dans l'émouvante mise en forme de l'orchestration de François Dompierre (Polydor 2912.034), partie du bottier Dans la mémoire longtemps.

4 On en retrouvera le texte à la page 144 de Cent chansons, Montréal, Fides, B.Q., 1988, 236 p. Cette réédition de 1970 a été précédée d'une introduction, p. 7 à 18, du soussigné : * Petits bonheurs du grand Félix chansonnier. On peut également consulter "Cent chansons de Félix Leclerc", Dictionnaire des œuvres litteraires du Québec, tome V, Montréal, Fides, 1987, p. 105-110. 
des sabots («agrandis en flaques d'eau»). Cette poétique des origines traduit déjà la quête amoureuse, ici malheureuse, car les signes permettant une lecture mémoriale ont été abolis. Le sentier a été déchiré par les labours. Dans les guérets, ne reste plus que le symbole de l'écriture, le bouleau, cet arbre que Marie-Victorin qualifie de déliquescent. Restent encore des symboles de mort, d'exil, de tristesse, toutes valeurs mises en valeur par la littérature canadienne-française ${ }^{5}$. Il y a bien, dans le deuxième couplet, une tentative de réfection de cet univers floué mais le poète, dans la troisième reprise du refrain, un refrain musicalement inchangé mais dont les mots se renouvellent, en appelle à l'oubli et à l'abolition de l'été.

Présence ${ }^{6}$, une grande chanson de 1948, reprendra autrement cette thématique. Le dialogue est alors simulé. Alors que le destinataire de la chanson de 1934 n'est évoqué que par le «notre » du titre et l'impératif final triplé: "Oublie l'été, oublie le jour / Oublie mon nom et le bouleau...", la chanson de 1948 joue davantage sur la construction d'un échange dialogué, celui de l'amante et de l'espoir contre l'incroyance de l'amant prostré qui se voit troublé par les sortilèges de l'autre. Par ailleurs, cette désespérance de l'homme se retrouve chez Bozo (1946), le marais ayant pris la forme liquide du labour: c'est non seulement le sentier "près du ruisseau " qui est brisé, c'est le ruisseau lui-même, devenu sans fil conducteur dans les eaux mortes. On pourrait multiplier ainsi les exemples pour montrer, dans la poésie sonorisée de Leclerc, l'alternance de la désespérance et de l'espoir. À ce titre, soulignons encore que Hymne au printemps (1949) reconstitue autrement, passant de l'automne au printemps, dans la même chanson et d'une chanson à l'autre, les mêmes éléments déjà en présence dans Notre sentier. Faut-il rappeler d'ailleurs que cette chanson, devenue après 1970 une chanson de liberration collective, n'est tout au plus, en 1949, qu'une chanson paysanne que les critiques n'étaient pas prêts de trouver belle, étant donnée la manière plutôt agacée dont ils accueillent la même année les Dialogues d'hommes et de bêtes.

Cette entrée en matière sur Notre sentier de Félix Leclerc m'apparaissait éclairer préalablement l'analyse du Tour de

5 C'est-à-dire une littérature marquée par la dualité, l'aliénation et la déréliction, une littérature de la perte du réel.

6 Cent chansons, p. 142. 
lîle que j'ai choisi de faire, la chanson éponyme de l'avantdernier microsillon de Leclerc ${ }^{7}$, l'une des plus souvent entendues malgré sa longueur d'écoute qui défie les radios industrielles, soit six minutes quarante ${ }^{8}$. Cette chanson s'arc-boute en quelque sorte sur Notre sentier tellement elle reprend, en l'affirmant, un espace d'appartenance, d'enracinement. La désespérance y est devenue affirmation sereine de soi.

On notera tout d'abord que la chanson est toute portée par la musique, introduite par elle et lui laissant toute la place en finale. Musique aérienne s'il en est une, toute portée par la harpe qui « touche les mains du vent *, * regarde les yeux du soleil», "chante la flûte de la nuit ${ }^{9}$. Musique circulaire par la redite, sorte de ritournelle prenant la solennité d'un rituel et qui assure protection en quelque sorte par sa forme circulaire, le début rejoignant la fin, prenant la forme ovoïde de l'île d'Orléans qui est évoquée (I-IV), provoquée (V), invoquée (VI-VII) ${ }^{10}$.

$\mathrm{Au}$ plan musical, cette reprise du thème introductif et final, puis entre les séquences trois et quatre, rapproche la chanson de l'eau, le ruisseau intime de 1934 devenu le fleuve de natalité, le sentier transformé en une île qui rappelle la cathédrale de Chartres, une cathédrale marine où la naissance mystérieuse s'est opérée, où la contradiction des signes pourraient pourtant advenir, où la vigie se fait l'oreille dressée, surveillant à la fois le golfe des secours séculaires attendus et Montréal qui donnera le signal convenu.

\section{Félix Leclerc, Le tour de l'île, Philips, 6325 242, 1975.}

8 L'auteur a eu l'occasion d'aborder cette question dans *... à Félix Lecterc, chantre fervent de la terre ", Les adieux du Québoc à Félix Leclerc, Québec, Les presses laurentiennes, 1989, p. 79-91; voir aussi L'action nationale, novembre $1988, p .775-785$. On peut aussi consulter a Félix Leclerc ou l'ile d'Orléans microcosme de la mémoire ", dans André Gaulin et Norbert Latulippe, L'île d'Orléans microcosme du Québec, publication par l'Association québecoise des professeurs de français, Québec, 1984, p. 127-131.

9 C'est le texte de l'unique refrain de la chanson fantaisiste et poétique malgré son prosaïsme, Sur la corde à linge, 1963.

10 A part quelques exceptions de chansons dont les textes sont parus dans des livres non publiés par Leclerc, les chansons datées d'après 1970 n'ont pas été éditées. Ce travail reste à faire. Cependant, Le tour de l'île est paru dans un grand livret du même titre, livre bellement illustré par Gilles Tibo aux éditions La courte échelle, a Montréal, en 1980, sans pagination. Nous partons de ce texte écrit, découpé en sept séquences et comptant 182 lignes. Le texte suit le découpage musical et inversement, introduit et terminé par des suites sonores, avec, entre la troisième et la quatrième partie, un ilot sonore qui marque un temps d'arrêt dans la chanson méditative. 
Faut-il le noter encore, musicalement, Le tour de líle ne comporte pas de refrain. Il s'agit plutôt de sept couplets dont le septième, légèrement détaché du sixième, n'est qu'une conclusion ouverte qui équivaut à sa manière aux derniers vers des Gens de mon pays de Vigneault: «Je vous entends demain/ Parler de liberté "11. Cela indique assez, dans ce type de chanson ${ }^{12}$, une démarche toute réflexive, intimiste en l'occurrence, qui commande une interprétation sobre, éventuellement lyrique dans sa forme itérative, que la récitante de Café Rimbaud II ${ }^{13}$ a mal saisi, me semble-t-il. En effet, Sylvie Legault a préféré une récitation déclaratoire, de ton stéréotypé, quand dans la séquence $\mathrm{V}$, l'île d'Orléans est proposée à la voracité d'une exploitation touristique aliénante. L'auteure qui réussit beaucoup mieux Mes blues passent pu dans 'porte de Pierre Huet - passe même à côté de l'image féminisée (qu'elle garde au masculin) de l'île vue comme «fleur de lyse». Cette prosopopée, presque, rejoint d'ailleurs la neige rose de février " comme chair de femme " 14 (v. 18, passage II).

La modalité du texte Le tour de lîle est par ailleurs indiquée d'entrée de jeu: «Pour supporter le difficile / et l'inutile" (v. 1-2). Ce difficile et cet inutile, liés grammaticalement, sont distancés et valorisés sur le plan sonore. L'un et l'autre pourraient paraître abstraits, mais sont nettement rattachés à une pièce dite, chose très rare chez Félix, qui précède immédiatement la chanson et que l'ensemble des enregistrements ont respectés, liant toujours Un an déjà à la chanson du Tour de líle ${ }^{15}$. Or, qu'évoque cette pièce que la seule copie enregistrée de 1975 accompagne déjà

11 Sorte de futur antérieur qui rejoint le constant futur antérieur du texte événementiel québécois: « Un jour j'aurai dit oui à ma naissance $*$, « Pour mon rapatriement ", dans L'homme rapaille de Gaston Miron.

12 On peut se rapporter à L'oiseau des vacances chez Charles Trenet, a Confession d'un malandrin chez Eduardo Branduardi, etc.

13 Café Rimbaud II, Sélect, 4.0010, cassette ou disque compact. Cette réalisation de Jean-François Doré et Jean-Pierre Paiement permet d'entendre, dites, interprétées sans musique, vingt chansons pour leurs seuls textes. On peut lire une présentation du soussigné dans le $n^{\circ} 76$ de Québec françals, hiver 1990, « Chanson et oralité ", p. 92-93.

14 On peut voir comme cette perception de février, chez Leclerc, inverse en quelque sorte la vision nelliganienne de * Soir d'hiver *, un Nelligan que Leclerc célèbre poette en son temps, comme Juif sous les nazis dans son dernier disque Mon fils, Polydor 2424 187, 1978 (pièce $\propto$ Nelligan " dont le texte figure en page 231 du Petit livre bleu de Félix).

15 Par exemple, Félix. Leclerc/Claude Léveillée Le temps d'une saison, Polydor, 2675 144; Félix Leclerc, Merci la France (album double), Philips, 
sonorement, en fondu, du thème musical de la chanson qui suit? La mort d'un ami cher permet à Félix de rappeler la bêtise sociale, certes, la vie publicitaire qui dégorge dans la vie privée, mais surtout la condition historique sous l'occupation anglaise, le vin troqué pour la bière prenant valeur de symbole.

Aussi l'île d'Orléans apparaît-elle à Félix comme un havre de repos et de paix, une halte dans le temps, une fuite dans l'espace «Pour supporter le difficile et l'inutile" (v. 1-2). Ce difficile vient, à n'en pas douter, du combat historique mené afin que la marée des siècles n'abolisse pas la fleur de lis historique: «Pour oublier grande blessure dessous l'armure » (v. 67), alors que surgit des îles de l'espace, «lîle d'Orléans / notre fleur de lyse " (v. 55-56). Lî̂le ressemble à un reposoir, un lieu de retraite au sens double du mot, au sens surtout de l'espace de mémoire qui permet de bondir à nouveau, les « clochers pointus » (v. 38) prenant même l'allure de l'épée du combat.

La chanson Le tour de lǐle est construite en tenant essentiellement compte de la durée. Les balises du temps, février, juillet, mai, été, hiver sont comme les flambeaux d'un rituel séculaire. Lî̀le perdure dans les marées hautes et basses, elle prend la proportion d'une cathédrale marine - « lîle c'est comme Chartres / [...] / avec des nefs / avec des arcs des corridors / et des falaises" (v. 11-15), sorte d'église de templiers où la mémoire veille et surveille: "et un grand-père au regard bleu / qui monte la garde» (v. 62-63). Cet homme de blanc et de bleu, aux couleurs de la France du Régime français occupée par l'Autre, est de connivence avec les éléments de la permanence, «Maisons de bois / maisons de pierre / clochers pointus " (v. 36-38) qui dardent dans le dur du temps et témoignent des origines ethno-culturelles d'une collectivité. Ce vieillard, à la lucidité née de l'amour mémorial, regarde constamment vers l'est, vers le golfe, vers le pays mythique de ses origines et de son ressourcement, cette douce France évoquée au début et à la fin de la chanson. France souveraine comme l'île habitée à force d'amour, de patience, de résistance, avec le constant retour saisonnier des oies sauvages qui n'oublient jamais la gestuelle du vol têtu qui l'emporte sur la pesanteur. Ainsi, « les descendants de La Rochelle/présents tout le temps / surtout

6679011 ; Félix Lederc, L'ancêtre, Kebec-disc, KD2 3008; Félix Leclerc, Le tour de l'île, Philips, 6325 242. Une seule exception: Johanne Blouin ne fait pas Un an déjà, mais joint au Tour de l'fle la chanson Notre sentier. 
54

l'hiver / comme des arbres » (v. 29-32) résistent, persistent, durent, perdurent et conjurent de faire l'indépendance, parce qu'à l'île, « quand on y pense / [...] / c'est comme en France * (v. 70-72). Lîle, symbolique du Québec, est vue comme pays.

Tibo, dans son illustration du texte, a retenu cette image de l'arbre, toujours présent, * surtout l'hiver * (v. 31) comme une image centrale du texte. On peut penser ici à cette image des Croquis laurentiens de Marie-Victorin qui, dans les « Trois chansons *, parle à propos des arbres, des « grands bras verriqueux ${ }^{16}$ de nos élans. L'illustrateur a même conçu l'arbre en groupe, en confrérie ${ }^{17}$, en établissant une homologie entre la cathédrale évoquée dans le texte et la nef impressionnante de la forêt orléanaise.

*Le difficile / et l'inutile* (v. 1-2), évoqués au début du texte de la chanson, se précisent avec la séquence où l'anglicisation menace la * fleur de lyse * (v. 56), cette autre langue, un autre décodage culturel, une utilisation même folklorique qui fasse d'un espace ovoïde, à la fois un dépotoir et un cimetière, «US parking " (v. 51) ose chanter "le fou de l'île *.

On ne peut, en un sens, concevoir chanson plus didactique - et sans qu'il y paraisse, d'où son grand art - de Félix Leclerc. Chanson circulaire, où la mélodie reprend inlassablement sa séquence pacifiante comme vague de marée montante, Félix s'adresse à ses compatriotes sceptiques qui croient peu ou prou que le ciel peut faillir, les arbres cesser de pousser. La chanson apparaît comme un texte destiné à l'homme colonisé, non convaincu de sa propre beauté et de celle de son pays - *Mais c'est pas vrai / ben oui c'est vrai / écoute encore " (v. 33-35) -, incrédule devant le scénario de la dépossession ( Imaginons », v. 45) et qui a besoin finalement du mage, du poète, fidèle à la mémoire d'un temps et d'un espace: "raconte encore» (v. 59). Plus que dans Notre sentier ou Présence, le dialogue est engagé, Leclerc sait maintenant qu'il a des destinataires. Il les connait et utilise son talent de conteur. Il imagine, avec eux, leur propre libération.

La fin de la chanson évite ainsi toute équivoque, Félix a su lire dans les signes de son pays: fruits mûrs dans les

16 Marie-Victorin, Croquis laurentiens, Montréal, Fides, 1985.

17 C'est Miron qui parle de la * Confrérie d'épinettes " dans * Les siecles de l'hiver ". 
vergers, quarante-deux milles de tour deviennent l'épanouissement de la mer et l'altière vision des montagnes. Il faut "célébrer l'indépendance " (v. 69): «l'heure est venue / si t'as compris..." (v. 81-82) La chanson se termine alors sur une longue séquence sonore qui se confond avec la marée saintlaurentienne. Un homme libre est né, rejoint par beaucoup d'autres, femmes et hommes, et qui poursuivent une longue naissance. Leur pays, issu d'un fleuve, est porté par une langue d'expression française: « c'est-y en France / c'est comme en France / le tour de l'île» (v. 71-73).

Dès lors, on peut même penser que le poète joue sur les mots : c'est au tour de l'île. Un tour à jouer. Sept tours à faire, que Jéricho tombe!

On pourrait certes écrire encore sur cette grande chanson sans en épuiser le sens, un sens resté ouvert parce que le destinataire n'a pas encore cru *l'heure [...] venue*. Une chanson toute faite d'un long travelling comme cette petite chanson de 1966, La vie. Un travelling dans le paysage, une remontée dans l'histoire descendue de La Rochelle, une simulation de l'abolition des signes communs et surtout une figuration de ce qui peut arriver à tout humain, l'histoire de sa libération.

Une chanson qui commande en quelque sorte son approche intertextuelle, une histoire qui se fixe en la chantant, chez Leclerc. Une histoire à intérioriser et dont la séquence musicale finale appelle plus la réflexion que les applaudissements. 